\title{
Simulation, MMSE Estimation, and Interpolation of Sampled Continuous-Time Signals Using Factor Graphs
}

\author{
Lukas Bolliger and Hans-Andrea Loeliger \\ ETH Zurich \\ Dept. of Information Technology \& Electr. Eng. \\ 8092 Zürich, Switzerland \\ Email: \{bolliger, loeliger\}@isi.ee.ethz.ch
}

\author{
Christian Vogel \\ Graz University of Technology \\ Signal Proc. \& Speech Comm. Lab. \\ 8010 Graz, Austria \\ Email:vogel@tugraz.at
}

\begin{abstract}
The paper proposes a factor graph representation of finite-dimensional continuous-time linear systems / filters driven by white Gaussian noise. Applications of such factor graphs include (i) simulation of the output signal at arbitrary discrete instants and (ii) MAP/MMSE/LMMSE estimation of the input signal, or of the state, or of the output signal at arbitrary discrete instants from noisy observations of the output signal at arbitrary discrete instants. Moreover, it is suggested that the MMSE estimate of the input signal from the output signal may be viewed as a generalization of a bandlimited estimate that is suited for real filters.
\end{abstract}

\section{INTRODUCTION}

In this paper, we propose a factor graph representation of continuous-time finite-dimensional linear systems driven by white Gaussian noise. Specifically, let $X \in \mathbb{R}^{n}$ be the state vector of such a system, which evolves in time according to

$$
\dot{X}(t)=A X(t)+B U(t)
$$

where $\dot{X}$ denotes the derivative with respect to time and where the real square matrix $A$ and the real vector $B$ are fixed and known. The real input signal $U(t)$ is assumed to be real-valued white Gaussian noise with autocorrelation function

$$
\mathrm{E}[U(t+\tau) U(t)]=\sigma_{U}^{2} \delta(\tau)
$$

where $\delta($.$) denotes the Dirac delta. We are interested in$ the relation between $X\left(t_{0}\right)$ and $X\left(t_{1}\right)$ for arbitrary $t_{0}$ and $t_{1}>t_{0}$. Note that, for a fixed initial state $X\left(t_{0}\right)=x\left(t_{0}\right)$, equation (1) induces a probability density $f\left(x\left(t_{1}\right) \mid x\left(t_{0}\right)\right)$ over the possible values of $X\left(t_{1}\right)$, which (unsurprisingly) turns out to be Gaussian.

The pivotal observation of this paper is that the function $f\left(x\left(t_{1}\right) \mid x\left(t_{0}\right)\right)$ can be used as a building block in discrete-time factor graphs. Discrete-time linear Gaussian factor graphs as in [1] are thus extended to include exact models of continuous-time systems of the form (1) between arbitrary discrete points in time. In consequence, we immediately obtain efficient algorithms for a variety of MAP/MMSE/LMMSE estimation problems involving noisy observations of continuous-time systems at arbitrary sampling instants. The underlying continuous-time system does not even need to be time invariant: time invariance is required only between discrete points in time.

We will describe the following applications:

- The simulation of discrete-time samples of $X\left(t_{k}\right)$ for arbitrary discrete sampling times $t_{0}, t_{1}, t_{2}, \ldots$.

- MAP/MMSE/LMMSE estimation of the state vector $X\left(t_{k}\right)$ for arbitrary instants $t_{0}, t_{1}, \ldots$ from noisy discretetime observations of $Y(t)=C X(t)$ (for some known row vector $C$ ) at arbitrary sampling times.

- Resampling of sampled filter output $Y(t)=C X(t)$ (for some known $C$ ) from and to arbitrary discrete-time sampling points.

- MAP/MMSE/LMMSE estimation of the input signal $U\left(t_{k}\right)$ for arbitrary instants $t_{0}, t_{1}, \ldots$ from noisy discretetime observations. It will be suggested that this estimate of the input signal may be viewed as a generalization of a bandlimited estimate of $U(t)$ that is suited for real filters.

We will use Forney-style factor graphs (normal factor graphs) as in [1] and [2]. In particular, we refer to the discussion of Gaussian message passing in [1].

The paper is structured as follows. The sum-product message computation rules through a node / factor $f\left(x\left(t_{1}\right) \mid x\left(t_{0}\right)\right)$ are given in Section II. The mentioned applications are discussed in Section III and some numerical examples are given in Section IV.

\section{Message Computation Rules}

The computation rules for the sum-product messages through a node / factor $f\left(x\left(t_{1}\right) \mid x\left(t_{0}\right)\right)$ as defined in Section I are given in Table I. All messages are Gaussian densities (up to a scale factor, which we ignore) and parameterized by the mean vector $\vec{m}$ and the covariance matrix $\vec{V}$ for forward messages or by $\overleftarrow{m}$ and $\overleftarrow{V}$ for backward messages.

The computation of the forward message amounts to integration of (1), which is standard [3]. The symmetry between the forward message and the backward message is obvious from the decomposition of $f\left(x\left(t_{1}\right) \mid x\left(t_{0}\right)\right)$ into $N$ discrete time steps as shown in Fig. 1. This decomposition is an 
TABLE I

COMPUTATION RULES FOR GAUSSIAN MESSAGES THROUGH NODE / FACTOR $f\left(x\left(t_{1}\right) \mid x\left(t_{0}\right)\right)$ WITH $t_{1}>t_{0}$.
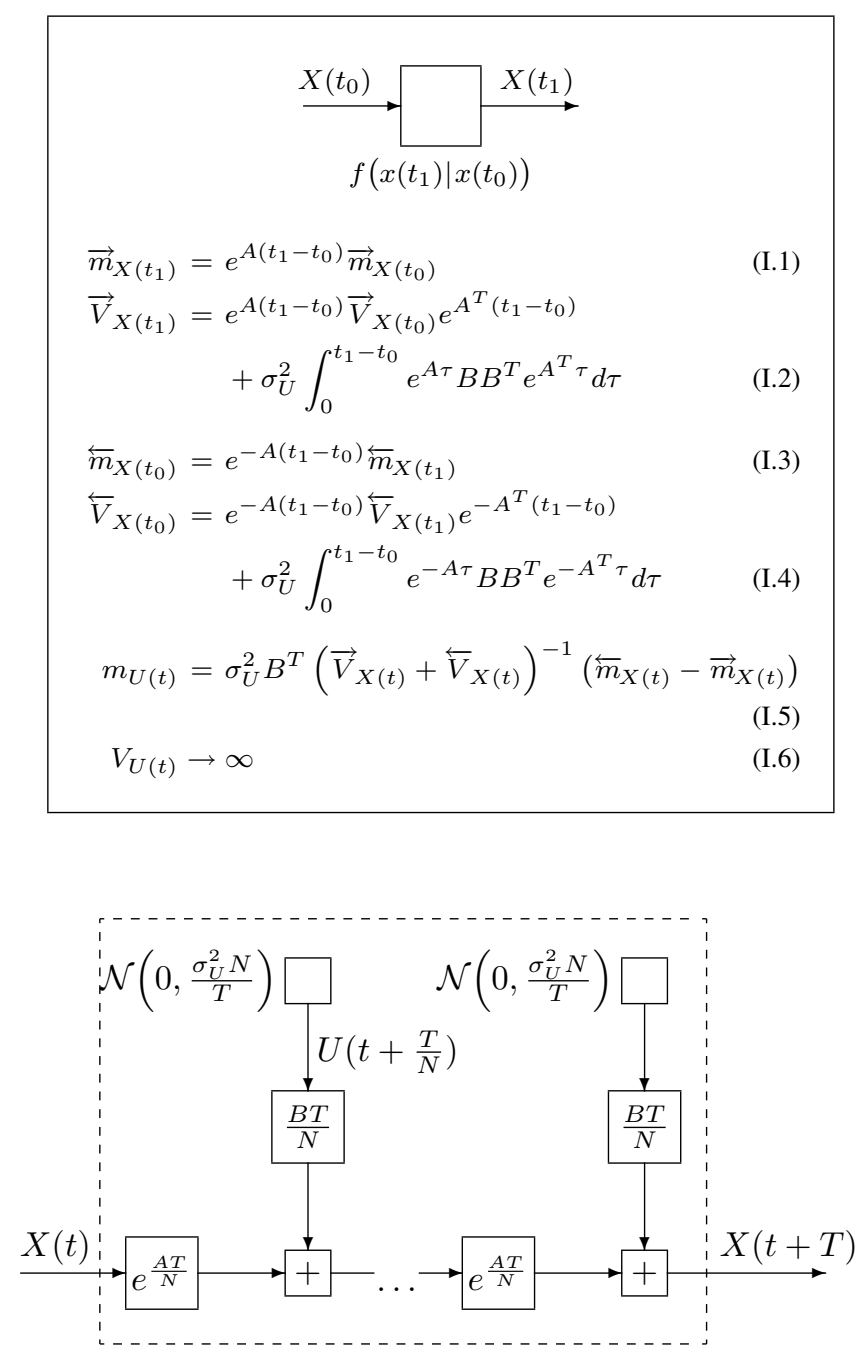

Fig. 1. Decomposition of the node / factor $f(x(t+T) \mid x(t))$ into $N$ discrete time steps. The figure is a factor graph as in [1] and $\mathcal{N}\left(m, \sigma^{2}\right)$ denotes a Gaussian probability density with mean $m$ and variance $\sigma^{2}$.

approximation which is exact in the limit $N \rightarrow \infty$. All claims in Table I may proved by computing the messages in Fig. 1 using the recipes of [1] and taking the limit $N \rightarrow \infty$.

A closed-form expression for the integral in (I.2) and (I.4) for the case where the matrix $A$ is diagonalizable over $\mathbb{C}$ is given in the appendix.

Of particular interest in Table I is (I.5), which is the mean of the marginal distribution of $U(t)$ for $t=t_{0}$ or $t=t_{1}$. If the overall factor graph is linear Gaussian and cycle free, $m_{U(t)}$ is the MAP/MMSE/LMMSE estimate of $U(t)$ [1]. Equation (I.6) reminds us that the variance of this estimate must be infinite because white noise cannot be fully estimated from discrete samples. Nevertheless, $m_{U(t)}$ can be a highly useful estimate as will be discussed in Section III-D.

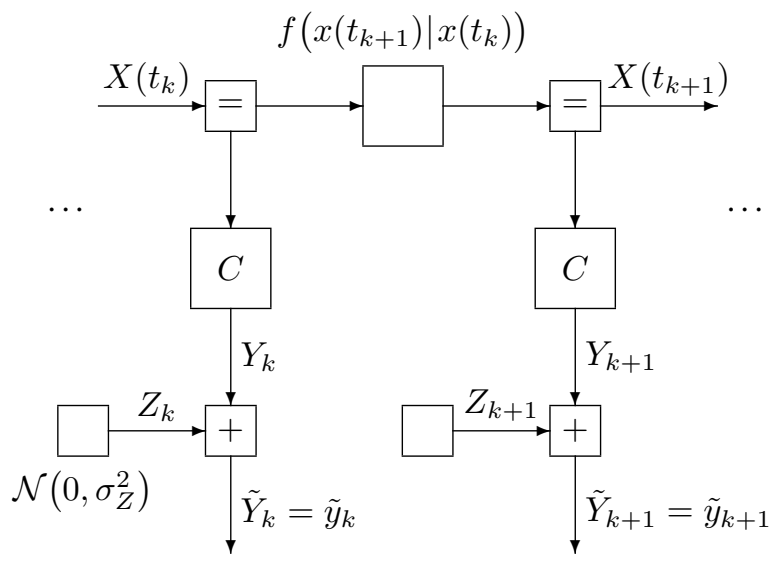

Fig. 2. Factor graph of continuous-time system with discrete-time observations $\tilde{Y}_{k}=\tilde{y}_{k}$.

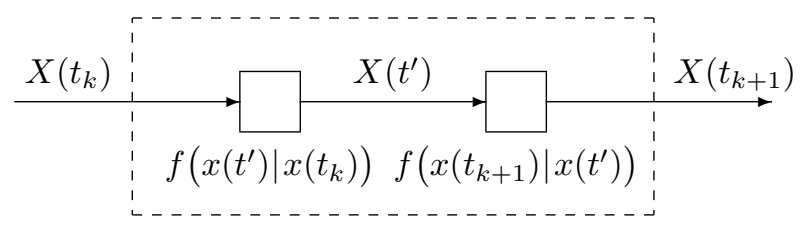

Fig. 3. Splitting the node/factor $f\left(x\left(t_{k+1}\right) \mid x\left(t_{k}\right)\right)$ to access the state at an intermediate point in time $t^{\prime}$

\section{APPLICATIONS}

\section{A. Simulation of Discrete-Time Samples of $X(t)$ and $Y(t)$}

Assume $t_{0}<t_{1}<t_{2}<\ldots$ Samples $x\left(t_{0}\right), x\left(t_{1}\right), x\left(t_{2}\right) \ldots$ of $X(t)$ can be obtained as follows. Assume we have sampled $X\left(t_{k}\right)=x\left(t_{k}\right)$. Conditioned on $X\left(t_{k}\right)=x\left(t_{k}\right)$, $X\left(t_{k+1}\right)$ is Gaussian with mean $\vec{m}_{X\left(t_{k+1}\right)}$ and covariance matrix $\vec{V}_{X\left(t_{k+1}\right)}$ computed according to (I.1) and (I.2) with $\vec{m}_{X\left(t_{k}\right)}=x\left(t_{k}\right)$ and $\vec{V}_{X\left(t_{k}\right)}=0$. From this distribution, we draw a sample $x\left(t_{k+1}\right)$, etc.

If the system has also an output $Y(t)=C X(t)$, we may easily obtain the corresponding samples $y\left(t_{k}\right)=C x\left(t_{k}\right)$. In this way, we can simulate discrete-time samples of filtered continuous-time white Gaussian noise.

\section{B. State Estimation from Discrete-Time Observations}

We now assume that, for discrete times $t_{k}$, we have observations

$$
\tilde{Y}_{k}=C X\left(t_{k}\right)+Z_{k}
$$

where $C$ is a known real row vector and where $Z_{1}, Z_{2}, \ldots$ is discrete-time white Gaussian noise with variance $\sigma_{Z}^{2}$. The factor graph of such a system model is shown in Fig. 2.

Since such a system is linear Gaussian, MAP / MMSE/LMMSE estimates of $X\left(t_{k}\right)$ may be obtained by Gaussian sum-product message passing in Fig. 2 as discussed in [1].

By splitting the node/factor $f\left(x\left(t_{k+1}\right) \mid x\left(t_{k}\right)\right)$ as shown in Fig. 3, such estimates may also be obtained for times $t^{\prime}$ between observations. 


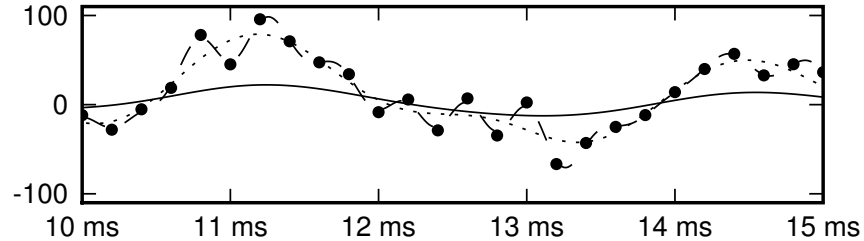

Fig. 4. Output signal estimation for different assumed values of $\sigma_{U}^{2} / \sigma_{Z}^{2}$. Dashed line: $\sigma_{U}^{2} / \sigma_{Z}^{2}=10$; dotted line: $\sigma_{U}^{2} / \sigma_{Z}^{2}=10^{-1}$; solid line: $\sigma_{U}^{2} / \sigma_{Z}^{2}=10^{-2}$.

\section{Output Signal Estimation, Interpolation, and Resampling}

It is straightforward to extend state estimation as in the previous section to estimating the noise-free output

$$
Y(t) \triangleq C X(t)
$$

at any fixed time $t$.

For any stochastic process $Y(t)$ representable as filtered white Gaussian noise, we may thus obtain estimates of $Y(t)$ at arbitrary points in time based on observations $\tilde{y}_{k}$ at arbitrary discrete times $t_{k}$. The Fourier spectrum of the estimate is controlled by the ratio $\sigma_{U}^{2} / \sigma_{Z}^{2}$ as will be discussed Section III-D.

\section{Input Signal Estimation}

Using (I.5), state estimation as in Section III-B may be extended to estimating the input signal $U(t)$ at arbitrary points in time. The estimated pair $(\hat{u}(t), \hat{x}(t))$ with $\hat{u}(t)=m_{U(t)}$ as in (I.5) minimizes

$$
\frac{1}{\sigma_{U}^{2}} \int u(t)^{2} d t+\frac{1}{\sigma_{Z}^{2}} \sum_{k}\left(\tilde{y}_{k}-C x\left(t_{k}\right)\right)^{2}
$$

subject to the constraints of the system model. The estimation thus prefers those frequencies in $U(t)$ that appear with little damping in $Y(t)=C X(t)$. The variance $\sigma_{U}^{2}$ may be viewed as a regularization parameter that controls the use of power in frequences outside the passband of the filter in order to fit the estimated output signal to the observations $\tilde{y}_{k}$.

The spectrum of $\hat{u}(t)$ is thus shaped by the spectrum of $Y(t)$, and the MMSE estimate $\hat{u}(t)$ may be viewed as a generalization of a bandlimited reconstruction that is suitable for real filters as opposed to the ideal lowpass filters of the sampling theorem.

\section{NUMERICAL EXAMPLES}

The continuous-time system in the following examples is a 4th-order Butterworth lowpass filter with a cut-off frequency of $f_{c}$. The discrete-time observations are regularly spaced and sampled at a rate of $f_{s}=10 f_{c}$, and they are subject to discretetime additive white Gaussian noise. The signal was simulated as described in Section III-A.

\section{A. Output Signal Estimaton}

The results of a numerical experiment are shown in Fig. 4. The fat dots show the noisy discrete-time observations $\tilde{y}_{k}$. The three lines show the interpolation as described in Section III-C for three different assumed values of $\sigma_{U}^{2} / \sigma_{Z}^{2}$.
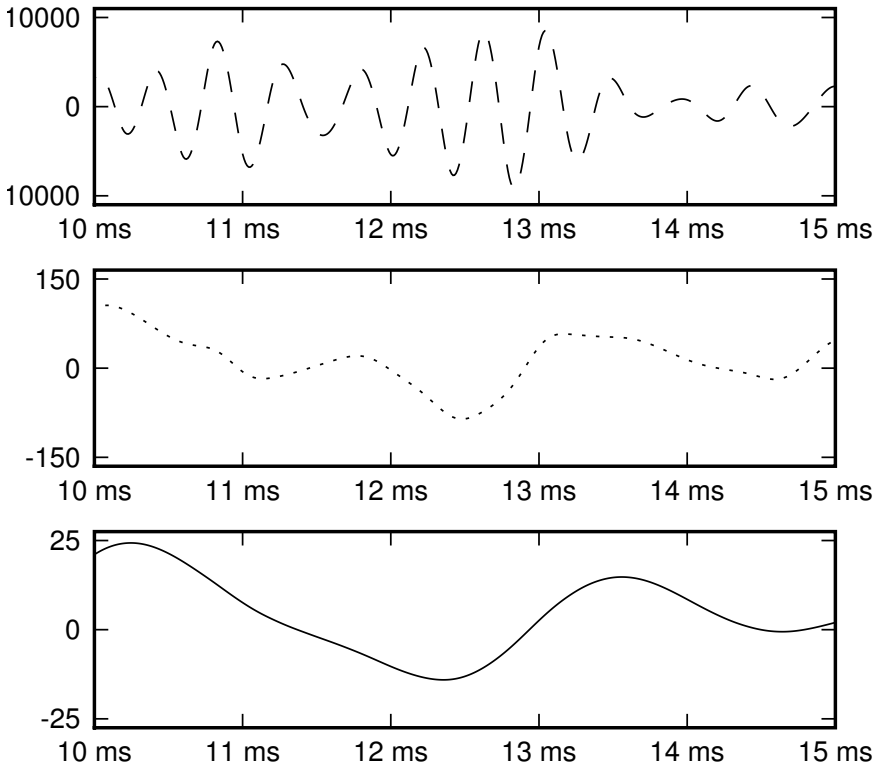

Fig. 5. Input signal estimation for the same discrete-time observations and the same three assumed values of $\sigma_{U}^{2} / \sigma_{Z}^{2}$ as in Fig. 4.

\section{B. Input Signal Estimation}

For the same discrete-time observations as in Fig. 4, estimating the input signal as described in Section III-D yields the results shown in Fig. 5. The different spectral content in the three plots illustrates the discussion in Section III-D.

\section{CONCLUSIONS}

We have pointed out that exact models of continous-time linear systems drived by white Gaussian noise can be used in discrete-time factor graphs. The associated continous-time signals thus become computational objects that can be handled with arbitrary temporal resolution by discrete-time Gaussian message passing.

We have also pointed out that the MMSE estimate of the input signal of such a system (based on discrete-time observations) is a continuous signal that may be viewed as a generalization of a bandlimited reconstruction that is suitable for real filters as opposed to the ideal lowpass filters of the sampling theorem.

\section{APPENDIX}

Assume that the matrix $A$ in (1) can be written as

$$
A=Q\left(\begin{array}{ccc}
\lambda_{1} & & 0 \\
& \ddots & \\
0 & & \lambda_{n}
\end{array}\right) Q^{-1}
$$

for some complex matrix $Q$. In this case, the integral in (I.2) and (I.4) can be solved in closed form:

$$
\int_{0}^{t} e^{A \tau} B B^{T} e^{A^{T} \tau} d \tau=Q(\Theta \odot(\Phi(t) \odot \Pi-\Pi)) Q^{H}
$$


and

$$
\int_{0}^{t} e^{-A \tau} B B^{T} e^{-A^{T} \tau} d \tau=Q(\Theta \odot(\Pi-\Phi(-t) \odot \Pi)) Q^{H}
$$

with

$$
\begin{aligned}
& \Theta \triangleq\left(\begin{array}{ccc}
\frac{1}{\lambda_{1}+\overline{\lambda_{1}}} & \cdots & \frac{1}{\lambda_{1}+\overline{\lambda_{N}}} \\
\vdots & \ddots & \\
\overline{1} & \cdots & \frac{1}{\lambda_{N}+\overline{\lambda_{N}}}
\end{array}\right) \\
& \Phi(t) \triangleq\left(\begin{array}{ccc}
e^{t\left(\lambda_{1}+\overline{\lambda_{1}}\right)} & \cdots & e^{t\left(\lambda_{1}+\overline{\lambda_{N}}\right)} \\
\vdots & \ddots & \\
e^{t\left(\lambda_{N}+\overline{\lambda_{1}}\right)} & \cdots & e^{t\left(\lambda_{N}+\overline{\lambda_{N}}\right)}
\end{array}\right) \\
& \Pi \triangleq Q^{-1} B\left(Q^{-1} B\right)^{H}
\end{aligned}
$$

where $\bar{x}$ denotes the complex conjugate of $x$, where $Q^{H} \triangleq \bar{Q}^{T}$ denotes the Hermitian transposition of $Q$, and where $\odot$ denotes component-wise multiplication.

\section{REFERENCES}

[1] H.-A. Loeliger, J. Dauwels, Junli Hu, S. Korl, Li Ping, and F. R. Kschischang, "The factor graph approach to model-based signal processing," Proceedings of the IEEE, vol. 95, no. 6, pp. 1295-1322, June 2007.

[2] H.-A. Loeliger, "An introduction to factor graphs," IEEE Signal Proc. Mag., Jan. 2004, pp. 28-41.

[3] H. Garnier and L. Wang, Identification of Continuous-Time Models from Sampled Data. Springer Verlag 2008. 